\title{
Actitudes frente al alcohol de los estudiantes de enfermería en dos universidades Latinoamericanas
}

\author{
Nursing students' attitudes towards alcohol \\ in two Latin-American universities
}

\section{Atitudes em relação ao álcool por estudantes de enfermagem em duas universidades latino-americanas}

\author{
A. Castañeda ${ }^{\mathrm{a} 1}$, L.P. Díaz-Herediab ${ }^{\mathrm{b} 2 *}$,V. Rodríguez ${ }^{\mathrm{c} 1}$, L. Penagos-Cubillos ${ }^{\mathrm{d} 1}$, C. Zambrano $^{\mathrm{e} 1}$, J. Castillo ${ }^{\mathrm{f3}}$ \\ ORCID: \\ ${ }^{a} 0000-0002-1882-6615$ \\ b $0000-0002-7167-282 \mathrm{X}$ \\ ${ }^{d} 0000-0002-2902-9400$ \\ e 0000-0003-2835-0822 \\ ${ }^{f} 0000-0001-8622-7908$ \\ ${ }^{1}$ Facultad de Enfermería, Universidad Nacional de Colombia, Bogotá D.C. Colombia \\ 2 Departamento de Enfermería, Facultad de Enfermería, Universidad Nacional de Colombia, Bogotá D.C., \\ Colombia \\ ${ }^{3}$ Escuela Nacional de Enfermería y Obstetricia, Universidad Nacional Autónoma de México, Ciudad de México, \\ México
}

Recibido: 19 febrero 2018

Aceptado: 11 septiembre 2018

\section{Resumen}

Objetivo: Comparar las actitudes de los estudiantes de enfermería de dos universidades: una de México y otra de Colombia, respecto al consumo de alcohol, el alcoholismo y los trastornos relacionados al abuso de este.

Método: Estudio cuantitativo comparativo transversal. Muestra de 231 estudiantes del último año de la carrera de enfermería en dos universidades públicas latinoamericanas; participantes voluntarios mayores de edad que cursaron al menos 6 asignaturas práctico-teóricas, a quienes se les aplicó el instrumento Escala de Actitudes Frente al Alcohol, el Alcoholismo y la persona con trastornos relacionados al uso del alcohol- EAFAA, así como una encuesta sociodemográfica y académica. Se utilizó SPSS 22 y Microsoft Excel ${ }^{\circledR}$ para el análisis estadístico y se tuvieron en cuenta los principios bioéticos. 
Resultados: La percepción de los estudiantes respecto a las personas con trastornos relacionados al uso de alcohol ( $\mathrm{p}=0.003$ ), es diferente según la universidad, lo cual conlleva una visión distinta con relación a su fuerza de voluntad, competencia de autocuidado, entre otras características. Asimismo, se determinó que la formación brinda herramientas al estudiante para afrontar situaciones de miedo, inseguridad, frustración, que favorecen un mejor cuidado $(\mathrm{p}=0.045)$.

Conclusiones: La presente investigación aporta al conocimiento de enfermería, ya que muestra en qué dimensiones la actitud puede afectar el trato brindado al paciente y cómo esta relación puede mejorarse a través de la formación en el área y de experiencias exitosas.

Palabras clave: Actitud; cuidados de enfermería; educación en enfermería; estudiantes de enfermería; abuso de alcohol; Colombia; México.

\section{Abstract}

Objective: To compare the attitudes of nursing students towards alcohol consumption, alcoholism, and alcohol-related diseases, in two universities: one in Mexico and the other in Colombia.

Method: This is a quantitative, comparative, and transversal study with a sample of 231 legal aged senior nursing students from two public universities of Latin-America who volunteered to participate. The Scale of Attitudes Towards Alcohol, Alcoholism, and Persons with Alcohol Related Diseases (SATAAP) instrument was administered in conjunction with a sociodemographic and academic survey. SPSS 22 and Excel were used to carry out the calculations. The corresponding bioethical principles were respected.

Results: The perception of students regarding to persons with alcohol-related diseases $(p=0.003)$ was not the same between the two universities, suggesting that differences in their views about strength of will and self-care competence, among other characteristics could be present. It was also found that the school formation gives the students tools to address situations of fear, insecurity, and frustration, thus fostering better self-care $(\mathrm{p}=0.045)$.

Conclusions: The present research builds on the nursing knowledge because it shows the importance of the attitude towards the use of alcohol, as well as its associated relationship between good school formation and better health care provided.

Keywords: Attitude; nursing care; education, nursing; students, nursing; abuse alcohol; Colombia; Mexico.

\section{Resumo}

Objetivo: Comparar atitudes sobre álcool, alcoolismo e pessoas com transtornos relacionados ao uso de álcool por estudantes de enfermagem sênior de duas universidades no México e na Colômbia.

Método: Estudo quantitativo comparativo transversal. Com amostra censo de 231 estudantes sênior de enfermagem de duas universidades públicas latino-americanas, participantes voluntários maiores de idade que cursaram pelo menos 6 disciplinas prático-teóricas, para os que foi aplicado o instrumento Escala de Atitudes frente ao Álcool, ao Alcoolismo e ao Alcoolista-EAFAA, e um questionário demográfico e acadêmico. SPSS 22 e Microsoft Excel ${ }^{\circledR}$ foram utilizados para análise estatística e tiveram-se em conta os princípios bioéticos de autonomia, justiça, beneficência e não maleficência.

Resultados: Encontrou-se diferenças entre as universidades sobre a percepção das pessoas com transtornos relacionados ao consumo de álcool $(\mathrm{p}=0,003)$, o que acarreta uma visão diferente em relação à sua força de vontade, competição pelo autocuidado, entre outras características. Da mesma forma, determinou-se que o treinamento proporciona ao aluno ferramentas para enfrentar situações de meda, insegurança, frustração, favorecendo um melhor cuidado $(\mathrm{p}=0.045)$.

Conclusões: Essa pesquisa contribui para o conhecimento de enfermagem, pois mostra em quais dimensões a atitude pode afetar o tratamento proporcionado ao paciente e como essa relação pode ser melhorada por medio de treinamento na área e experiências bem-sucedidas.

Palavras chave: Atitude; cuidados de enfermagem; educação em enfermagem; estudantes de enfermagem; abuso de álcool; Colômbia; México. 


\section{Introducción}

El consumo de alcohol es una de las grandes problemáticas de la salud pública que se presenta en la actualidad, esto se ve reflejado en los indicadores de morbilidad y mortalidad mundial reportados por la Organización Mundial de la Salud (OMS) en 2014, en los que se señala que cada año aproximadamente se producen 3.3 millones de muertes a nivel mundial, como consecuencia del consumo nocivo de alcohol, esto representa un 5.9\% de todas las defunciones en general. Además, la OMS señala que el consumo nocivo de alcohol es un agente causal de más de 200 enfermedades, principalmente crónicas no transmisibles, tales como cirrosis hepática, algunos tipos de cáncer y enfermedades cardiovasculares, esto sin dejar de lado los trastornos comportamentales, así como de traumatismos como consecuencia de la violencia y accidentes de tránsito ocasionados por personas en estado de embriaguez ${ }^{1}$.

En Colombia, según el Estudio Nacional de Consumo de Sustancias Psicoactivas del año 2013, 87\% de los encuestados declaró consumir alcohol alguna vez en su vida (consumo en hombres del $91 \%$ y 83\% en mujeres), el 35.8\% ingirió alcohol en los últimos 30 días (8.3 millones de personas, 46.2\% hombres y $25.9 \%$ mujeres), lo cual refleja en este contexto, que el consumo es algo habitual, esta percepción de normalidad predispone a la ingesta nociva y posteriormente a enfermedades derivadas de esta o al agravamiento de situaciones clínicas preexistentes; en relación con la edad, la mayor tasa de uso de alcohol se presenta en jóvenes entre los 18 y los 24 años (proporción del 49.2\%), seguido del grupo entre 25 y 34 años (proporción del 45.4\%). Asimismo, se estima que 2.5 millones de colombianos presentan un consumo perjudicial de bebidas alcohólicas de los cuales 1.9 millones son hombres y 0.6 millones son mujeres².

En México, se evidencia un aumento en el consumo excesivo de alcohol, de acuerdo con la Encuesta Nacional de Consumo de Drogas, Alcohol y Tabaco (ENCODAT, 2017), la edad de inicio de consumo para el 2016 se concentró entre los 18 y 25 años (41.3\%), la prevalencia de consumo alguna vez en la vida es $77.3 \%$, consumo en el último año es $53.3 \%$; éstas se han mantenido estables con respecto al 2011 , que presentaba $77.1 \%$ y $55.7 \%$, respectivamente; sin embargo, la prevalencia de consumo en el último mes aumentó de $35 \%$ a $39.9 \%$ y el patrón de consumo excesivo de alcohol en el último mes reporta un incremento entre el 2011 y el 2016, que paso de $13.9 \%$ a 22.1\%, en los hombres aumentó de $24.1 \%$ a $34.4 \%$; en las mujeres de $4.5 \%$ a $10.8 \%{ }^{3}$.

En relación a la atención de esta población, se estima que a pesar de la gravedad y el impacto mundial, aun cuando el consumo de alcohol está presente en el $20 \%$ a $50 \%$ de pacientes hospitalizados, sólo son diagnosticados un $5 \%$, por tanto, el profesional de enfermería puede estar gran parte del tiempo al cuidado de personas con consumo nocivo de alcohol, sin tener conocimiento de ello, o sin asumirlo como una problemática importante ${ }^{4}$.

La presente investigación surge al considerar por una parte, la epidemiología del consumo de alcohol y por otra, el comportamiento del personal de enfermería con este tipo de población, y se plantea como objetivo principal comparar las actitudes, en relación al consumo de alcohol, el alcoholismo y hacia las personas con trastornos relacionados al abuso de alcohol; de estudiantes de enfermería inscritos en el último año de la carrera en universidades públicas en México y Colombia.

El principio teórico principal es que la construcción de actitudes se fundamenta en creencias y concepciones, así como en las experiencias y formación académica, con base en los hallazgos de la literatura y las propuestas teóricas, la formación termina por ser una variable predictora de la futura conducta de los profesionales ${ }^{5-9}$; los factores culturales también pueden influir en esta construcción ${ }^{10}$.

La comparación entre países puede ser de gran utilidad, ya que permite vislumbrar la influencia de la formación en contextos diferentes, además de que esta variable es susceptible de modificación para mejorar la atención a las personas consumidoras de alcohol y los trastornos relacionados a éste. Por otra parte, la caracterización y comparación del fenómeno en estudio en países latinoamericanos es necesaria, debido a que los estudios existentes en el área, en su mayoría se concentran en Brasil, por tanto se debe 
conocer y analizar en otros países, para observar su comportamiento y generar bases para estudios de intervención ${ }^{11}$.

Para determinar el tipo de actitud que tienen los estudiantes de enfermería frente al fenómeno del alcoholismo, es necesario analizar las diferencias existentes con respecto a las de los estudiantes de otras áreas, donde el contexto, los conocimientos y las experiencias previas, permiten identificar cómo se van generando y caracterizando las actitudes en los estudiantes de enfermería. Este hecho se evidencia en un estudio realizado en Croacia, en el cual se comparan las actitudes frente al alcoholismo en estudiantes de enfermería y mecatrónica, donde los primeros presentaron actitudes positivas hacia las personas con alcoholismo, mientras que los segundos actitudes más positivas hacia el consumo de alcohol. Se concluyó que aspectos tales como el ambiente, la aceptación social y los conocimientos sobre los diversos efectos del alcoholismo en la salud y el comportamiento, tuvieron una repercusión en las actitudes de los participantes ${ }^{12}$.

Así, investigar cómo son las actitudes de los estudiantes de enfermería en dos universidades latinoamericanas y si estas difieren entre países, permitiría entrever cómo se dará la relación terapéutica entre el profesional de enfermería y el paciente con problemas asociados al consumo nocivo de alcohol, ya que las actitudes son un componente fundamental para la toma de decisiones ${ }^{13}$, en este caso, decisiones de cuidado, de acuerdo a estas, el enfermero se decidirá por una conducta evasiva o por realizar acciones de intervención, que permitan una atención integral orientada a la prevención de nuevos episodios ${ }^{8,14-21}$, de ahí surge la pregunta ¿Son diferentes las actitudes sobre el consumo de alcohol, el alcoholismo y hacia las personas con trastornos relacionados al abuso de alcohol, en estudiantes de enfermería del último año de dos universidades, una de México y otra de Colombia? Con el propósito de identificar prácticas formadoras de actitudes positivas que se pueden mantener y desencadenantes de actitudes negativas que se pueden mitigar en las universidades.

\section{Método}

Se realizó un estudio cuantitativo comparativo transversal, la muestra se integró por estudiantes de enfermería matriculados en el último año de la carrera en dos universidades latinoamericanas públicas, una de Colombia y otra de México en 2016. Se utilizó para la recolección de la información el instrumento Escala de Atitudes frente ao Álcool, ao Alcoolismo e ao Alcoolista-EAFAA, versión español para América Latina ${ }^{11}$, herramienta que se originó en idioma portugués ${ }^{22}$ y debido a la alta población en América Latina cuyo idioma oficial es el español, se adaptó la escala a este idioma, se obtuvo así un instrumento fiable con un índice de validación de contenido de 0.97 , un índice de kappa 0.80 y un índice de correlación interclases de 0.92 . Su adaptación cultural se desarrolló en Colombia ${ }^{11}$. Cabe señalar que se desarrolló una validación aparente en el contexto mexicano para su uso en este estudio, a fin de asegurar la comprensión de la información contenida en el instrumento por la población.

La muestra estuvo constituida por 231 estudiantes de enfermería: 100 estudiantes pertenecientes a la universidad colombiana de una población de 135 y 131 estudiantes de la universidad mexicana de una población de 201. Estos representan el $74.1 \%$ y $65.2 \%$ de respuesta, respectivamente.

Los criterios de inclusión del estudio fueron aceptar participar voluntariamente en el estudio, ser mayor de edad, haber contestado en su totalidad el instrumento y ser estudiante de último año de enfermería; este criterio fue fundamental, ya que se consideró que ellos tendrían un mayor conocimiento y experiencia en el trabajo con personas que consumen alcohol. En relación a los criterios de exclusión se tuvo en cuenta a aquellas personas que no hubieran completado el instrumento de manera correcta o que no fueran parte de la población a estudiar.

La recolección de los datos contó con dos etapas principales, la primera fue llevada a cabo en México durante el primer semestre del año 2016 y la segunda recolección en Colombia durante el segundo semestre del mismo año. En cada universidad, al inicio se obtuvo el aval por parte del comité de ética, posteriormente se realizó una búsqueda institucional de los estudiantes que cursaban el último año de 
la carrera, se les expuso el propósito de la investigación, se aclararon dudas, conforme a ello resolvían participar o no voluntariamente, en seguida se entregaba y se recibía el consentimiento informado en un sobre, separado del instrumento y la encuesta de datos sociodemográficos y académicos a desarrollar, esto para proteger la identidad de las personas que participaron.

La variable evaluada a través del instrumento es la actitud, entendida esta como la Posición de una persona sobre una dimensión bipolar evaluativa 0 afectiva con respecto de un objeto, acción 0 evento y representa el sentimiento favorable 0 desfavorable de una persona hacia un objeto o estímulo ${ }^{5}$. La evaluación se realiza mediante una escala tipo Likert de cinco puntos donde los estudiantes expresan su opinión sobre cada afirmación, según el grado de concordancia que perciben de cada enunciado, cinco es lo más positivo. Esta escala define un punto de corte de 3.2 para establecer si es una actitud positiva $(\geq 3.2)$ o negativa $(<3.2)^{11}$.

El instrumento utilizado: Escala de Actitudes Frente al Alcohol, el Alcoholismo y la persona con trastornos relacionados al uso del alcohol-EAFAA, consta de 49 ítems distribuidos en 4 dimensiones ${ }^{11}$ :

1. El trabajo y las relaciones interpersonales con pacientes con trastornos relacionados al uso de alcohol. Evalúa si el estudiante experimenta sentimientos de inseguridad, miedo, apatía para el trabajo con personas con consumo nocivo de alcohol, ya que esto dificulta la relación terapéutica. Incluye 17 ítems relacionados a la percepción, opiniones, sentimientos y actitudes relativas a brindar cuidado en salud a pacientes con trastornos relacionados al uso del alcohol.

2. La persona con trastornos relacionados al uso de alcohol. Características que atribuye el estudiante a la persona con consumo nocivo de alcohol, como el irrespeto, irresponsabilidad, carácter débil, falta de autocuidado y adherencia al tratamiento. Abarca 13 ítems referentes a las concepciones, percepciones, opiniones y actitudes relativas al paciente con trastornos relacionados al uso de alcohol. Los ítems expresan concepciones sobre las características propias de esa población.

3. El alcoholismo (etiología). Posibles causas del alcoholismo según el estudiante, como problemas familiares, sociales, psicológicos. Agrupa 10 ítems relativos a las percepciones sobre las motivaciones y causas para el uso de alcohol y el alcoholismo; se basa en la explicación bio-psicosocial para los trastornos relacionados al uso de alcohol y abarca ítems relativos a factores psíquicos, sociales, biológicos y morales; los cuales son atribuidos como causa del uso de alcohol y de los trastornos relacionados.

4. Las bebidas alcohólicas y su uso. Concepción del estudiante sobre el consumo de alcohol, puede verse como algo normal, como un derecho o una decisión personal, o puede pensar que se debe beber con moderación y tener control personal del consumo. Reúne nueve ítems que hacen referencia a opiniones y actitudes relativas a las bebidas alcohólicas y el derecho de las personas a beber.

Para el análisis estadístico de la información recolectada, se realizó una base de datos en los programas Microsoft Excel ${ }^{\circledR}$ y SPSS 22. Para comparar las cuatro dimensiones y el total del instrumento EAFAA en los dos países, se aplicó la prueba de U de Mann Whitney ${ }^{23}$.

Se siguieron los principios de justicia, autonomía, beneficencia y no maleficencia y se contó con los avales de los Comités de Ética de las respectivas instituciones educativas, se diligenció un consentimiento con cada uno de los participantes ${ }^{24}$. La investigación salvaguardó la identidad de los participantes, quienes contestaron el instrumento de manera anónima.

\section{Resultados}

Las características sociodemográficas de los dos grupos de Colombia y México dejan ver en ambos grupos el predominio de las mujeres con $67 \%$, y $61 \%$, respectivamente, el estado civil mayoritario fue el de soltero con $91 \%$ y $87 \%$ y la edad promedio de ambos grupos fue de 23 años. Se realizó una comparación entre los grupos, se confirmó que eran similares en cuanto a sus características sociodemográficas; posteriormente se evalúo si las variables de estudio presentaban una distribución normal a través de la prueba de Kolmogórov Smirnov ${ }^{23}$, se determinó que las dimensiones 1, 3 y 4 no la tuvieron, se rechazó 
entonces la hipótesis de normalidad de la distribución, debido a esta razón se decidió utilizar estadística no paramétrica para los análisis posteriores.

En la Tabla 1a, es posible apreciar que en el total de la escala y en tres de las cuatro dimensiones, en ambos países, se obtuvieron promedios que indican actitudes positivas ( $\geq 3.2)$, sólo en la dimensión 3 relativa a etiología del alcoholismo el promedio fue menor; en cuanto a las características de la persona con trastornos relacionados al uso de alcohol la diferencia de promedios alcanzó significancia estadística $(\mathrm{p}=0.003)$, aunque ambos promedios reflejan actitudes positivas, el promedio de los estudiantes de Colombia es mayor. Esta dimensión abarca ítems referentes a las concepciones, percepciones, opiniones y actitudes de los estudiantes relativos a la persona con trastornos relacionados al uso de alcohol, asuntos que están muy influenciados por la cultura del lugar donde se vive, por lo tanto, en cada país los estudiantes perciben a la persona de manera diferente en relación a atributos como la responsabilidad, fuerza de voluntad, competencias de autocuidado, adherencia al tratamiento y la capacidad de relacionarse.

Se realizó la comparación de las dimensiones del instrumento EAFAA entre hombres, así como entre mujeres según el país, no hubo diferencias estadísticamente significativas, es decir que los estudiantes de enfermería en ambos países, independientemente de si son hombres o mujeres, tienen actitudes similares frente a las bebidas alcohólicas, el alcoholismo o las personas con trastornos relacionados al uso de alcohol en el momento de la atención. Sin embargo, en un segundo análisis desarrollado por cada país según sexo, sólo en los estudiantes de Colombia (Tabla 1b), se encontró una diferencia estadísticamente significativa ( $\mathrm{p}=0.025$ ) entre hombres y mujeres en la cuarta dimensión (bebidas alcohólicas y su uso), específicamente en la pregunta 16 del instrumento que corresponde a ¿beber alcohol en cualquier cantidad causará siempre dependencia? Los hombres presentan un mejor promedio que las mujeres, lo que refleja una actitud de mayor aceptación en el uso/consumo de alcohol.

Tabla 1. Promedios por dimensiones y total de las actitudes de los estudiantes de enfermería en la Escala EAFAA y, según sexo de estudiantes de Colombia

\begin{tabular}{|c|c|c|c|c|c|c|c|}
\hline & & \multicolumn{3}{|c|}{ 1a. Promedio total } & \multicolumn{3}{|c|}{ 1b. Sexo (Colombia) } \\
\hline & Dimensiones del instrumento EAFAA & Colombia & México & $\mathbf{P}$ & Hombre & Mujer & $\mathbf{P}$ \\
\hline 1 & $\begin{array}{l}\text { El trabajo y las relaciones interpersonales con pacientes } \\
\text { con trastornos relacionados al uso de alcohol }\end{array}$ & 3.6 & 3.6 & 0.544 & 3.6 & 3.6 & 0.883 \\
\hline 2 & $\begin{array}{l}\text { La persona con trastornos } \\
\text { relacionados al uso de alcohol }\end{array}$ & 3.5 & 3.3 & $.003 *$ & 3.4 & 3.6 & 0.331 \\
\hline 3 & El alcoholismo (etiología) & 2.8 & 2.8 & 0.568 & 2.8 & 2.8 & 0.368 \\
\hline 4 & Las bebidas alcohólicas y su uso & 3.3 & 3.3 & 0.904 & 3.5 & 3.3 & $.025 *$ \\
\hline 5 & Todas las dimensiones & 3.3 & 3.3 & 0.093 & 3.3 & 3.3 & 0.634 \\
\hline
\end{tabular}

En la comparación entre México y Colombia respecto a la pregunta de la encuesta sociodemográfica y académica $¿$ Ha recibido preparación para trabajar con personas que presentan trastornos relacionados al uso de alcohol y otras drogas? se encontraron diferencias estadísticamente significativas en la primera dimensión trabajo y las relaciones interpersonales con pacientes con trastornos relacionados al uso de alcohol $(\mathrm{p}=0.045)$ (Tabla 2), quienes han recibido formación en el área tienen mejores actitudes de acuerdo a sus promedios, en cambio este promedio disminuye en quienes no han recibido dicha formación. Esta dimensión abarca las percepciones, opiniones, sentimientos y actitudes relativas a brindar cuidado en salud a pacientes con trastornos relacionados al uso de alcohol. Esta diferencia permite observar la relación existente entre la formación y cómo el estudiante se relaciona con la persona con consumo nocivo de alcohol, en donde la formación le ayuda al estudiante a disminuir el miedo, la inseguridad, la frustración y sentimientos negativos, y lo capacita para brindar un mejor cuidado y establecer una relación terapéutica, habilidades que no podrá desarrollar cuando la formación es limitada o nula. 
Tabla 2. Promedios en las dimensiones del instrumento EAFAA respecto a las Actitudes de los estudiantes de enfermería según formación

\begin{tabular}{|c|c|c|c|c|c|c|}
\hline & \multirow{3}{*}{ Dimensiones del instrumento EAFAA } & \multicolumn{5}{|c|}{ Formación en el área de alcohol } \\
\hline & & \multicolumn{2}{|c|}{ Colombia } & \multicolumn{2}{|c|}{ México } & \multirow{2}{*}{$\mathbf{P}$} \\
\hline & & Si & No & Si & No & \\
\hline 1 & $\begin{array}{l}\text { El trabajo y las relaciones interpersonales con pacientes } \\
\text { con trastornos relacionados al uso de alcohol }\end{array}$ & 3.6 & 3.5 & 3.6 & 3.4 & $.045^{*}$ \\
\hline 2 & La persona con trastornos relacionados al uso de alcohol & 3.6 & 3.4 & 3.3 & 3.3 & 0.152 \\
\hline 3 & El alcoholismo (etiología) & 2.8 & 2.8 & 2.8 & 2.8 & 0.534 \\
\hline 4 & Las bebidas alcohólicas y su uso & 3.3 & 3.4 & 3.4 & 3.3 & 0.684 \\
\hline 5 & Todas las dimensiones & 3.3 & 3.3 & 3.3 & 3.2 & $.037 *$ \\
\hline
\end{tabular}

En la Tabla 3 se identifica que para los estudiantes de México que participaron en la investigación, hay una diferencia estadísticamente significativa $(\mathrm{p}=0.034)$, entre la pregunta de la encuesta sociodemográfica y académica ¿El alcoholismo es una enfermedad? y la cuarta dimensión Las bebidas alcohólicas y su uso, que hace referencia a opiniones y actitudes relativas a las bebidas alcohólicas y el derecho de las personas a beber, esto puede deberse a que los conceptos propios del derecho a beber, ve la bebida como algo normal, apreciación de beneficio o perjuicio para la salud y el control personal, sobre su uso se relacionan directamente con la percepción del alcoholismo como una enfermedad autoinflingida, que está mediada por la fuerza de voluntad y el control de la persona sobre el consumo. De manera comparativa, para los estudiantes de Colombia que participaron, hay una diferencia estadísticamente significativa $(\mathrm{p}=0.032)$ en esta misma pregunta ¿El alcoholismo es una enfermedad? con la segunda dimensión correspondiente a La persona con trastornos relacionados al uso de alcohol, que hace referencia a las concepciones, percepciones, opiniones y actitudes relativas a la persona con trastornos relacionados al uso de alcohol, es decir, los estudiantes de Colombia difieren si el alcoholismo es una enfermedad o no, relacionándolo con las características de la persona con consumo nocivo de alcohol, como la responsabilidad, fuerza de voluntad, autocuidado, recaídas, adherencia al tratamiento y capacidad de relacionarse con otros. En los cuatro constrastes de variables que se presentan, el promedio más bajo y que corresponde a mala actitud, se presentó en la $3^{\text {a }}$ dimensión referida a etiología del alcoholismo.

Además de los análisis anteriores también se realizó la comparación de la pregunta académica ¿Ha tenido experiencia con personas con trastornos relacionados al uso del alcohol? en donde se observa que para ambos países la actitud cambia si han tenido o no experiencia, pero no se identificaron diferencias estadísticamente significativas entre los países.

Tabla 3. Actitudes de los estudiantes de enfermería según Promedio en las dimensiones del instrumento EAFAA según reconocimiento del alcoholismo como enfermedad

Reconocimiento del alcoholismo como una enfermedad

\begin{tabular}{|c|c|c|c|c|c|c|c|}
\hline & \multirow[t]{2}{*}{ Dimensiones del instrumento EAFAA } & \multicolumn{3}{|c|}{ Colombia } & \multicolumn{3}{|c|}{ México } \\
\hline & & Si & No & $\mathbf{P}$ & Si & No & $\mathbf{P}$ \\
\hline 1 & $\begin{array}{l}\text { El trabajo y las relaciones interpersonales con pacientes } \\
\text { con trastornos relacionados al uso de alcohol }\end{array}$ & 3.6 & 3.6 & 0.415 & 3.6 & 3.6 & 0.604 \\
\hline 2 & La persona con trastornos relacionados al uso de alcohol & 3.5 & 3.8 & $.032 *$ & 3.3 & 3.4 & 0.365 \\
\hline 3 & El alcoholismo (etiología) & 2.8 & 2.8 & 0.567 & 2.8 & 2.9 & 0.956 \\
\hline 4 & Las bebidas alcohólicas y su uso & 3.3 & 3.5 & 0.648 & 3.4 & 3.0 & $.034^{*}$ \\
\hline 5 & Todas las dimensiones & 3.3 & 3.4 & 0.175 & 3.3 & 3.2 & 0.846 \\
\hline
\end{tabular}




\section{Discusión}

Las actitudes de los estudiantes entre los países evaluados mostraron diferencias en la segunda dimensión, estos resultados se explican, ya que las actitudes varían considerablemente por el contexto de desarrollo social y cultural entre las dos poblaciones. Este tema ha sido abarcado en diferentes estudios como en el artículo Estoy sola: la experiencia de las enfermeras en el cuidado del usuario de alcohol y drogas, donde a través de un estudio fenomenológico caracterizan a la persona que abusa del consumo de alcohol y es percibida como un usuario difícil, que justifica el consumo buscando pasar dificultades, evadir la realidad y calmar la angustia, lo catalogan en que disfruta el momento y pone poco esfuerzo para su recuperación; estas concepciones varían en función del grupo estudiado, de la formación y experiencias previas en el tema ${ }^{25}$.

Se encontró que para los estudiantes de Colombia hay una diferencia significativa entre hombres y mujeres, específicamente en la pregunta 16 que corresponde a ¿Beber alcohol en cualquier cantidad causará siempre dependencia?, los varones tienen actitudes más positivas que indican una tendencia más permisiva hacia el consumo de alcohol, esto puede deberse a que el consumo de sustancias psicoactivas es mayor en hombres de 18 a 24 años en la población colombiana, según el reporte de drogas de Colombia 2016, lo cual puede llevarlos a tener una conducta de mayor aceptación en comparación con las mujeres ${ }^{26}$; estos resultados concuerdan con el estudio realizado por Rocha et al., con estudiantes de pregrado de enfermería en el cual encontraron que el 55\% de estos ve el consumo de alcohol como algo normal, y 54\% están en desacuerdo de que cualquier cantidad de alcohol puede causar dependencia, sin embargo, no hace una especificación por sexo de esta variable. Los hallazgos en el grupo colombiano, probablemente pueden atribuirse a creencias culturales de la población masculina de una normalidad en el consumo que no causa dependencia. Es importante tener en cuenta que las creencias culturales previas son un componente importante en la formación de actitudes ${ }^{27}$.

En las siguientes dimensiones no se encontraron diferencias estadísticamente significativas entre los dos países, no obstante, se rescatan aspectos importantes al realizar el análisis de las preguntas sociodemográficas y académicas y su relación con las dimensiones. Por ejemplo en la pregunta ¿Ha recibido preparación para trabajar con personas con trastornos relacionados al uso de alcohol y otras drogas? se relacionó con la primera dimensión "trabajo y las relaciones interpersonales con pacientes con trastornos relacionados al uso de alcohol”. Este hecho refleja que existe una diferencia entre la repercusión que tiene el nivel de formación entre los dos países y que se evidencia en la relación que se genera entre el estudiante de enfermería y los pacientes con este tipo de trastornos. Lo que concuerda con el estudio de Vásquez y Pillon, en 2005 en Colombia, concluyen que la formación es fundamental para brindar atención a las personas con problemas de alcoholismo ${ }^{28}$.

Las experiencias en cuanto a la relación entre la formación y desempeño con los pacientes en el estudio efectuado por Rocha et al., en estudiantes de pregrado de enfermería, revela que hay un predominio de neutralidad y actitudes negativas relacionadas con la formación frente a este tipo de pacientes ${ }^{27}$, aunque ello difiere del contexto en el que se desarrolla la investigación. La formación y preparación de los estudiantes de enfermería, para el abordaje de los pacientes con trastornos relacionados al alcohol es un aspecto fundamental y necesario para que se logre generar una actitud positiva, con la cual se alcancé una interacción y un cuidado adecuado, tal como es propuesto en estudios realizados por Vargas et al., en $2010^{29}$, donde se estudia esta problemática, que revelan la gran necesidad y repercusión de los niveles de formación en el cuidado.

Para México, hay una diferencia estadísticamente significativa en relación de la pregunta sociodemográfica ¿El alcoholismo es una enfermedad? con la cuarta dimensión correspondiente a Las bebidas alcohólicas y su uso: que hace referencia a opiniones y actitudes relativas a las bebidas alcohólicas y el derecho de las personas a beber. Para Colombia, la diferencia se encontró en la misma pregunta, sin embargo, se relacionó con la segunda dimensión correspondiente a La persona con trastornos relacionados al uso de alcohol: que hace referencia a las concepciones, percepciones, opiniones y actitudes relativas al paciente con trastornos relacionados al uso de alcohol. 
De lo anterior se puede discernir que el grupo de estudiantes de México relacionan el hecho de enfermedad con el libre albedrío de cada quien, para beber en exceso o no, por tanto, pueden conceptualizar el alcoholismo como una enfermedad autoinfligida, esta concepción tendrá una gran repercusión en el cuidado, ya que pueden verla como una enfermedad evitable y se buscará el cuidado del cuerpo en esencia y dar tratamiento solamente a los síntomas físicos.

Para el grupo colombiano si el alcoholismo es una enfermedad o no, se relaciona con el carácter y la personalidad de quien consume, con la dimensión psicológica, puede ser positivo a la hora de brindar el cuidado, además tomar en cuenta esta dimensión e intervenir en ella, o negativo si por el contrario se piensa que por tener un componente tan profundo en la conducta humana estas personas no se pueden recuperar, o que no se cuenta con las herramientas terapéuticas necesarias para ayudar a la persona.

Vargas et al. en el 2010, relatan en su estudio debido a la formación médica que tienen los enfermeros, los cuidados brindados a este tipo de pacientes muchas veces se dirigen a controlar los síntomas físicos dejan de lado el componente psicológico, esto discrepa con los hallazgos en Colombia, ya que el $85 \%$ de este grupo consideran el alcoholismo una enfermedad, pero lo relacionan en gran medida con el carácter, personalidad y aspectos mentales de la persona ${ }^{30}$.

Finalmente, el que los promedios más bajos para las actitudes de los estudiantes de ambos países se ubiquen en la dimensión de la etiología del alcoholismo, indica una necesidad apremiante de mayor formación en esta área.

\section{Conclusiones}

Un mayor nivel de formación en el campo de atención a las personas con trastornos relacionados al uso de alcohol contribuye a la generación de actitudes más positivas ante estos pacientes.

Trabajar con las actitudes del personal de salud es un reto, pues puede determinar el actuar del profesional en un escenario, por tanto, es necesario enfatizar en una formación de calidad que brinde herramientas a los estudiantes para que se desempeñen adecuadamente en el mundo laboral.

Dentro de las limitaciones del estudio se encuentra la baja disponibilidad para responder a la invitación de los estudiantes por compromisos académicos y personales, lo cual se refleja en el porcentaje de respuesta.

La presente investigación aporta al conocimiento de enfermería debido a que muestra en qué dimensiones la actitud puede afectar el trato brindado al paciente, cómo esta relación puede mejorarse a través de la formación en el área y de experiencias exitosas. Da algunos elementos de juicio de cómo se está ofreciendo la formación en el área del profesional actualmente en dos universidades de América Latina, ya que es un pilar para proponer la inclusión de formación en el área para los estudiantes de enfermería. Asimismo, el presente estudio puede ser base de otros estudios comparativos sobre las actitudes de los estudiantes frente al alcohol, el alcoholismo y las personas con trastornos relacionados al uso de alcohol para reafirmar o reconsiderar los resultados obtenidos.

Por otra parte, gracias a la epidemiología del consumo de alcohol, se puede denotar que cualquier profesional de enfermería puede encontrarse en su práctica cotidiana con una persona con problemas de consumo de alcohol, esta investigación ratifica la necesidad de reconocer las particularidades que cada persona trae consigo al acto de cuidar; el brindar cuidados de forma integral, atender las necesidades físicas, emocionales, espirituales y demás que no estén satisfechas en este grupo de personas, puede ser modulado a través de la educación y los procesos formativos, así como la cultura de la cual se procede.

\section{Responsabilidades éticas}

Protección de personas y animales. Los autores declaran que para esta investigación no se han realizado experimentos en seres humanos ni en animales.

Confidencialidad de los datos. Declaran que en este artículo no aparecen datos de estudiantes. 
Derecho a la privacidad y consentimiento informado. Los autores han obtenido el consentimiento informado de los sujetos referidos en el artículo.

Financiamiento: Convocatoria para proyectos de investigación de la Vicerrectoría de Investigación sede de la Universidad Nacional de Colombia, al macro proyecto Alcohol y otras drogas: consumo actitudes y desafíos. Convocatoria 2016-2017 del que se originó este estudio.

Conflicto de intereses. Los autores declaran no tener conflicto de intereses

\section{Referencias}

1. World Health Organization. Global status report on alcohol and health. Luxemburgo: World Health Organization; 2014. [Consultado septiembre 13 2016]. Disponible en: https://bit.ly/2sHtA20

2. MinJusticia/MinSalud/UNODC/CICAD/INL. Estudio Nacional de Consumo de Sustancias Psicoactivas en Colombia. Bogotá D.C.: Observatorio de Drogas de Colombia (ODC); 2014. [Consultado agosto 18 2017]. Disponible en: https://bit.ly/1KA2m2o

3. INPRFM/INSP/CONADIC/SSA. Encuesta Nacional de Consumo de Drogas, Alcohol y Tabaco 20162017: Reporte de Alcohol. Ciudad de México: Secretaría de Salud; 2017. [Consultado septiembre 9 2018]. Disponible en: https://bit.ly/2rC9Tsm

4. MinSalud/Colciencias. Guía de Práctica Clínica para la detección temprana, diagnóstico y tratamiento de la fase aguda de intoxicación de pacientes con abuso o dependencia del alcohol. Bogotá D.C.: MinSalud; 2013. [Consultado septiembre 9 2018]. Disponible en: https://bit.ly/2Ds2WjO

5. Ajzen I, Fisbbein M. Factors influencing intentions and the intention-behavior relation. Hum Relat. 1974; 27(1): 1-15. https://doi.org/10.1177/001872677402700101

6. Ajzen I, Fishbein M. Understanding attitudes and predicting social behavior. Englewood Cliffs, NJ: Prentice-Hall; 1980.

7. López-Mena N. Creencias y actitudes de los estudiantes de enfermería ante el paciente que consume alcohol y el consumo de alcohol [Tesis]. Monterrey, Nuevo León: Facultad de Enfermería, UANL; 2012.

8. Crothers CE, Dorrian J. Determinants of Nurses' Attitudes toward the Care of Patients with Alcohol Problems. ISRN Nurs. 2011; 821514; p. 11 https://dx.doi.org/10.5402/2011/821514

9. Costa-Martins ER, Gollner-Zeitoune RC, Ribeiro-Francisco MTR, Spindola T, Bertolossi-Marta C. Concepções do trabalhador de enfermagem sobre drogas: a visibilidade dos riscos. Rev. Enferm. UERJ. 2009; 17 (3): 368-72. [Consultado septiembre 9 2017] Disponible en: https://bit.ly/2TanUJt

10. Agic B, Mann RE, Kobus-Matthews M. Alcohol use in seven ethnic communities in Ontario: A qualitative investigation. Drugs (Abingdon Engl): 2011; 18(2): 116-23. https://doi.org/10.3109/09687630903514909

11. León-Ramírez EG, Vargas D. Adaptação cultural e validação da escala de atitudes frente ao álcool, ao alcoolismo e ao alcoolista EAFAA versão espanhol para América Latina [Tesis]. São Paulo, Brasil: Escola de Enfermagem, Universidade de São Paulo; 2015.

12. Malčić T, Kudumija-Slijepčević M. Differences in the knowledge and attitudes between students of technical and nursing studies towards alcohol and alcoholism. Alcoholism and Psychiatry Research. 2015 [Consultado enero 26 2018]; 51(1): 15-30. Disponible en: https://bit.ly/2U9viVu

13. Aristizábal-Hoyos GP, Blanco-Borjas DM, Sánchez-Ramos A, Ostiguín-Meléndez RM. El modelo de promoción de la salud de Nola Pender. Una reflexión en torno a su comprensión. Enferm. univ. 2011; 8(4): 16-23. https://doi.org/10.22201/eneo.23958421e.2011.4.248

14. Vargas D. Nursing students' attitudes towards alcohol, alcoholism and alcoholics: a study of a brazilian sample. J Nurs Educ Pract. 2012; 2(1): 1-8. http://dx.doi.org/10.5430/jnep.v2n1p1

15. Telumbre-Terreno JY, Esparza-Almanza SE, Alonso-Castillo BA, Alonso-Castillo MTJ. Actitudes hacia el consumo de alcohol y consumo de alcohol en estudiantes de enfermería. Investig. enferm 2017; 19(2): 69-81. http://dx.doi.org/10.11144/Javeriana.ie19-2.ahca 
16. Mateo-Crisóstomo Y, Armendáriz-García NA, Alonso-Castillo MT, Martínez-Maldonado R. Conocimientos y creencias sobre el cuidado al usuario de alcohol por estudiantes de enfermería. Rev Cuid. 2016; 7(2): 1255-61. http://dx.doi.org/10.15649/cuidarte.v7i2.314.

17. Ford R, Bammer G, Becker N. Improving nurses' therapeutic attitude to patients who use illicit drugs: Workplace drug and alcohol education is not enough. Int J Nurs Pract. 2009; 15(2): 112-8. https: / doi.org/10.1111/j.1440-172X.2009.01732.x

18. López-Alabarce JA, Hernández-Sánchez D, Molina-Mula J, Fernández-Medina JM, Castaño-Fernández AI, Sanz-Álvarez E, et al. Percepción, conocimientos y actitudes de las enfermeras de urgencias y salud mental frente al alcoholismo y otras drogodependencias. Metas enferm 2014 [Consultado septiembre 9 2017]; 17(2): 22-31. Disponible en: https://bit.ly/2UfFP1D

19. Pillon SC, Ramos-Laranjeira R. Formal education and nurses' attitudes towards alcohol and alcoholism in a Brazilian sample. Sao Paulo Med J. 2005; 123(4): 175-80. http://dx.doi.org/10.1590/S1516-31802005000400004

20. Vargas D, Bittencourt MN. Álcool e alcoolismo: atitudes de estudantes de enfermagem. Rev. bras. enferm. 2013; 66(1): 84-9. http://dx.doi.org/10.1590/S0034-71672013000100013

21. Carraro TE, Hussein-Rassool G, Villar-Luis MAV. A formação do enfermeiro e o fenômeno das drogas no Sul do Brasil: atitudes e crenças dos estudantes de enfermagem sobre o cuidado. Rev. Lat.-Am. Enferm. 2005; 13(spe): 863-71. http://dx.doi.org/10.1590/S0104-11692005000700014

22. Vargas D, Villar-Luis M. Development and validation of scale of attitudes towards alcohol, alcoholism and alcoholics. Rev. Lat.-Am. Enferm. 2008. 16(5): 895-902.

http://dx.doi.org/10.1590/S0104-11692008000500016

23. Gibbons JD. Nonparametric Statistics: An Introduction/ Series on Quantitative Applications in the Social Sciences. Newbury Park, CA: Sage Publications, Inc; 1993.

24. Acevedo I. Aspectos Éticos en la Investigación Científica. Cienc. enferm. 2002. 8(1): 15-18. http: / /dx.doi.org/10.4067/S0717-95532002000100003

25. Ortega LB, Ventura CA. I am alone: the experience of nurses delivering care to alcohol and drug users. Rev Esc Enferm USP. 2013; 47(6): 1381-88. http://dx.doi.org/10.1590/S0080-623420130000600019

26. MinJusticia Reporte de Drogas de Colombia 2016. Bogotá D.C.: Observatorio de Drogas de Colombia (ODC); 2016 p.23.

27. Mota-Rocha F, Vargas D, Ferreira- de Oliveira MA, Bittencourt MN. Caring for people with psychoactive substance dependence: nursing student perceptions. Rev Esc Enferm USP. $2013 ; 47$ (3): 671-77. http://dx.doi.org/10.1590/S0080-623420130000300021

28. Vásquez-Mendoza E, Pillon SC. La formación de enfermeras y el fenómeno de las drogas en Colombia: conocimientos, actitudes y creencias. Rev. Lat.-Am. Enferm. 2005; 13 (n.spe): 845-53. http://dx.doi.org/10.1590/S0104-11692005000700012

29. Vargas-Vilela M, Arena-Ventura CA, da Silva EC. Conocimiento de estudiantes de enfermería sobre alcohol y drogas. Rev. Lat.-Am. Enferm. 2010. 18(n.spe): 529-34. http://dx.doi.org/10.1590/S0104-11692010000700007

30. Vargas D, Ferreira-de Oliveira MA, Villar-Luís MA. Care of alcoholic persons in primary care services: perceptions and actions of registered nursing. Acta Paul. Enferm. 201 0; 23(1): 73-9. http://dx.doi.org/10.1590/S0103-21002010000100012 